Document downloaded from:

http://hdl.handle.net/10251/124074

This paper must be cited as:

Pereira, J.; Colomer, A.; Naranjo Ornedo, V. (2018). Comparison of Local Analysis Strategies for Exudate Detection in Fundus Images. En Intelligent Data Engineering and Automated Learning - IDEAL 2018. Springer. 174-183. https://doi.org/10.1007/978-3-03003493-1_19

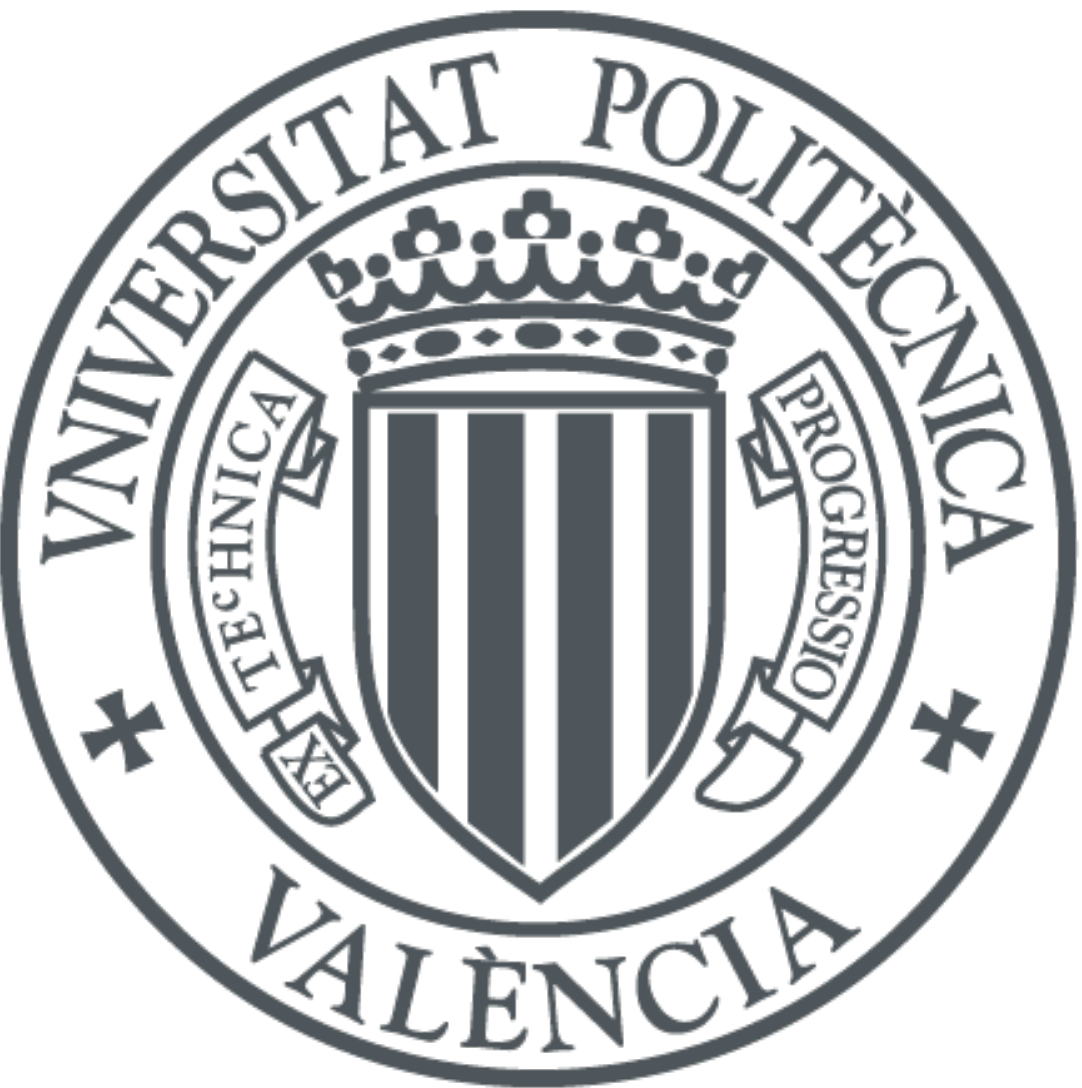

The final publication is available at

http://dx.doi.org/10.1007/978-3-030-03493-1_19

Copyright Springer

Additional Information 


\title{
Comparison of Local Analysis Strategies for Exudate Detection in Fundus Images
}

\author{
Joana Pereira ${ }^{1}$, Adrián Colomer ${ }^{2}$, and Valery Naranjo ${ }^{2}$ \\ ${ }^{1}$ University of Minho, Campus Gualtar, Braga 4710, Portugal \\ ${ }^{2}$ Instituto de Investigación e Innovación en Bioingeniería (I3B), \\ Universitat Politècnica de València, Camino de Vera s/n, 46022, Valencia, Spain
}

\begin{abstract}
Diabetic Retinopathy (DR) is a severe and widely spread eye disease. Exudates are one of the most prevalent signs during the early stage of DR and an early detection of these lesions is vital to prevent the patient's blindness. Hence, detection of exudates is an important diagnostic task of DR, in which computer assistance may play a major role. In this paper, a system based on local feature extraction and Support Vector Machine (SVM) classification is used to develop and compare different strategies for automated detection of exudates. The main novelty of this work is allowing the detection of exudates using non-regular regions to perform the local feature extraction. To accomplish this objective, different methods for generating superpixels are applied to the fundus images of E-OPHTA database and texture and morphological features are extracted for each of the resulting regions. An exhaustive comparison among the proposed methods is also carried out.
\end{abstract}

Keywords: Exudates · Superpixels · LBP · Granulometries · SVM.

\section{Introduction}

Diabetic Retinopathy (DR) is a common complication of diabetes, which is among the major causes of vision loss in the world. However, at the initial phase of the disease, the vision impairment is not easily realized by the patient $[1,2]$. Exudates are one of the most prevalent signs during the early stage of DR. These lesions are formed due to the leakage of blood and its early detection can improve patients' ${ }^{\prime}$ chances to avoid blindness [3]. In Fig.1 it is possible to observe the difference between a healthy fundus image and a retinal image containing exudates as a consequence of DR.

Manual detection of exudates by ophthalmologists is laborious and timeconsuming. Therefore, automated screening techniques for exudate detection have great significance in saving cost, time and labour, allowing the ophthalmologists to make the treatment decision timely [4]. In this sense, one of the main objectives of this work is to develop and compare different strategies to locally extract information of fundus images for detecting exudates.

Several methods related to the automatic detection of exudates have been proposed in the literature, these can be grouped in: thresholding-based $[5,6]$, 


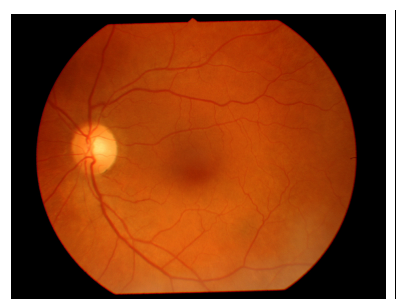

(a)

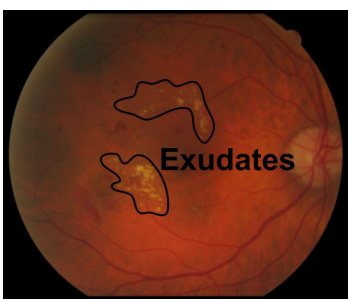

(b)

Fig. 1. Fundus images. (a) Healthy eye, (b) Pathological eye with exudates.

region growing-based $[3,7]$ and morphological-based classification $[6,8]$. These methods focus their efforts in the exudates segmentation, however, these approaches are characterised by presenting a high false-positive rate. For this reason, in the methodology proposed in this work, the characterisation of healthy and damaged retinal areas is performed by applying image descriptors in a local way, avoiding the segmentation step. Methods involving feature extraction and classification of the retinal tissue have been studied in the literature. The most common procedure requires the extraction of features from a lesion candidate map generated by different techniques, such as: mathematical morphology $[4,6]$; background subtraction [9]; clustering [10]; or using banks of filters and applying a low adaptive threshold $[11,12]$. Subsequently, the candidates are classified as exudate or non-exudate elements, making use of the extracted features and classification algorithms.

The strategies proposed in this work do not require the previous segmentation of exudates or the generation of candidate maps. Exudates usually represent less than one percent of the total number of pixels that compose the retinal image. For this reason, in the methodology presented in this paper, the image is divided in regions or patches and, during the feature extraction step, features vectores are extracted for each region. Different methods to generate superpixels are presented as a strategy to create non-regular regions.

Superpixels are regions resulting from a low-level segmentation of an image and are typically used as primitives for further analysis such as detection, segmentation, and classification of objects. The underlying idea is that this first low-level partition decreases the computational complexity of the following processing steps and improves their robustness [13]. Methods for generating superpixels have been studied in the literature. Those can be broadly categorized as: graph-based algorithms [14,15]; gradient ascent methods [16, 17]; or clustering methods [18]. Of these, the latter has particular interest since the Simple Linear Iterative Clustering (SLIC) algorithm [18], which is an adaptation of $k$-means for superpixel generation, will play an important role during the evaluation of this work. V. Machairas et al. [19] also proposed a strategy to create superpixels by applying the watershed transformation to a spatially regularized gradient to achieve a tunable trade-off between superpixels regularity and adherence to object boundaries. With this approach, the term "waterpixels" was introduced in 
the literature. The strategies proposed in this paper rely on the marker-controlled watershed transformation, in order to efficiently generate waterpixels.

The main objective of this work is to develop and compare different strategies to obtain regions of interest with the purpose of locally describing healthy and pathological retinal areas. Allowing the detection of exudates using nonregular regions to perform the local feature extraction is the main novelty of this work. Therefore, two different strategies for generating waterpixels are applied to the images of the E-OPHTA database. Subsequently, texture and morphological features are locally extracted and, finally, each region is classified according to healthy and pathological classes, during the classification stage. In the end, an elaborated comparison between the proposed strategies for generating waterpixels and the SLIC superpixels is also performed.

\section{Methods}

This paper's methodology allows the characterisation of healthy and damaged retinal areas by applying image descriptors in a local way. Therefore, it is possible to summarise the methodology proposed into three principal steps: computation of waterpixels; local feature extraction; and, finally, classification of the retinal tissue. These steps will be developed in the following subsections.

\subsection{Computation of Waterpixels}

Creation of the grid and gradient definition. The first step consists in computing a grid of regular cells $C_{i}$. A grid of hexagons is created with the size of the input image. Note that the hexagon side $s$ is a tunable parameter. The smaller $s$ is, the smaller the resulting waterpixels and the higher the number of resulting regions. The distance between adjacent hexagon centres is denoted as $\sigma$ and it plays a normalization role in the waterpixel computation process.

At this stage, the computation of the morphological gradient $g$ from the input image $f: D$ (where $D$ is a rectangular subset of $Z^{2}$ ) is performed. The morphological gradient can be defined as:

$$
g(f)=\delta_{B}(f)-\epsilon_{B}(f)
$$

where $\delta$ and $\epsilon$ are, respectively, the operators dilation and erosion and $B$ is a unitary structuring element.

Selection of the Markers. The selection of markers allows us to control the number and regularity of the resulting waterpixels: the number of markers is equal to the number of waterpixels in the final partition; to obtain regions which are similar in shapes and sizes, it is necessary to select the markers in a way that they are regularly spaced out over the image.

Taking this into account, a unique marker per cell is selected to obtain total control over the number of waterpixels, and a strong impact on their size and shape. During this procedure, it is necessary to have in mind that the ideal is to find a marker that enables to obtain the best performance in terms of boundary 
adherence and regularity. Therefore, two approaches are tested: selecting the centres of the grid cells as markers (c-Waterpixels) and selecting one minimum of the gradient per cell as markers (m-Waterpixels). In the second approach, each cell $C_{i}$ of the grid defines a region of interest where the content of $g$ is analysed to select a unique marker, taking into account the following considerations:

- if there exist more than one minimum of $g$ inside $C_{i}$, the one with the highest surface extinction value [13] is selected.

- if there is no minimum of $g$ inside $C_{i}$, the centre of $C_{i}$ is defined as marker (to guarantee regularity).

- if there is a unique minimum of $g$ inside $C_{i}$, this minimum is obviously selected as marker.

Spatial regularisation of the gradient and Watershed computation. A spatially regularised gradient $g_{r e g}$ is computed to guarantee a compromise between boundary adherence and regularity:

$$
g_{\text {reg }}=g+k d_{Q}
$$

where $g$ is the gradient of the image, $k$ is the spatial regularisation parameter, which, in this work, is set to one. Finally, let $Q=q_{i_{1<i<N}}$ be a set of $N$ connected components of the image $f$. For all $p \in D$, we can define a distance $d_{Q}$ with respect $Q$ as follows:

$$
\forall p \in D, d_{Q}(p)=\frac{2}{\sigma} \min _{i \in[1, N]} d\left(p, q_{i}\right)
$$

where $\sigma$ is the grid step defined in the previous section.

Finally, the watershed transformation is performed on the spatially regularised gradient $g_{r e g}$, starting the flooding from the markers. This allowed the partition of the image into waterpixels regions. Figure 2 shows the the resulting $\mathrm{c}$ and m-Waterpixels and Fig.3 illustrate the described steps to create this regions.

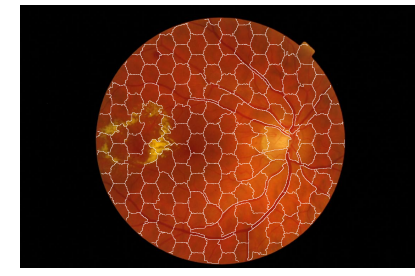

(a)

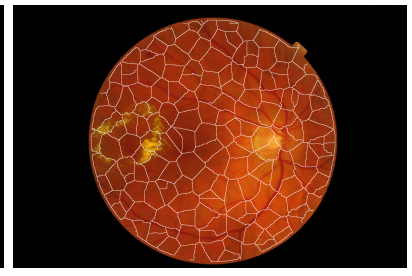

(b)

Fig. 2. Comparison of the two proposed strategies to generate waterpixels. (a) cWaterpixels and (b) m-Waterpixels.

\subsection{Feature Extraction}

Local Binary Pattern (LBP) is a simple yet very efficient texture descriptor which labels the pixels of an image by thresholding the neighborhood of each pixel: 

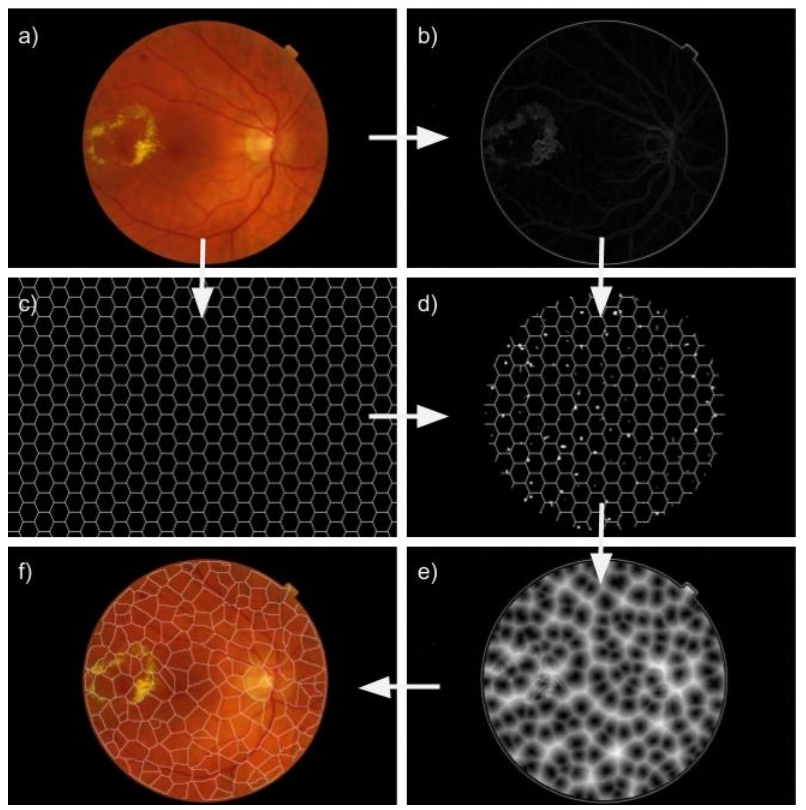

Fig. 3. Illustration of m-Waterpixels generation. (a) Original image, (b) Morphological gradient of the original image, (c) Regular grid of hexagonal cells $(s=32)$, (d) Selected markers within the regular grid, (e) Spatially regularised gradient, (f) m-Waterpixels.

$$
L B P_{P, R}=\sum_{p=0}^{P-1} s\left(g_{p}-g_{c}\right) \cdot 2^{p}, s(x)=\left\{\begin{array}{l}
1 \text { if } x \geq 0 \\
0 \text { if } x<0
\end{array}\right.
$$

where $P$ represents the number of samples on the symmetric circular neighbourhood of radius $R, g_{c}$ is the grey value of the pixel $(i, j)$ and $g_{p}$ is the grey value of each neighbour.

When LBP is used for texture description it is common to include a contrast measure by defining the Rotational Invariant Local Variance (VAR) as:

$$
V A R_{P, R}=\frac{1}{P} \sum_{p=0}^{P-1}\left(g_{p}-\mu\right)^{2}, \quad \mu=\frac{1}{P} \sum_{p=0}^{P-1} g_{p}
$$

$L B P_{P, R}^{r i u 2}$, defined in [20], and $V A R_{P, R}$ are complementary measures and the combination of both is expected to be a powerful descriptor for detecting abnormal retinal patterns. $L B P_{P, R}^{r i u 2}$ and $V A R_{P, R}$ operators are locally computed for each pixel of the green channel of the retinal image. As a result, LBP and VAR images are obtained. These resulting images are divided into waterpixels regions and normalised histograms are computed for each region combining the information provided by both images using the method proposed in [21]. 
Granulometries. Mathematical morphology has become increasingly relevant in the image processing field essentially due to its versatility and rigorous mathematical description. Granulometry is one of the most interesting techniques based on mathematical morphology.

Let $f$ be a grey-level image, $f \in F(E, T)$ such that $f(x): E \rightarrow T$ where $x=$ $(x, y) \in E$ is the pixel position and $E \subseteq \mathbb{Z}^{2}$. T is an ordered set of grey-levels. After the selection of a fixed set $B \subseteq E$, the two elementary operations of erosion $\left(\epsilon_{B}(X)\right)$ and dilation $\left(\delta_{B}(X)\right)$ can be composed together to generate a new set of grey-level operators given by the grey-level opening $\left(\gamma_{B}\right)$ and closing $\left(\varphi_{B}\right)$ :

$$
\begin{aligned}
& \gamma_{B}(f)(x)=(f \circ B)(x)=\delta_{B}\left(\epsilon_{B}(f)\right)(x) \\
& \varphi_{B}(f)(x)=(f \bullet B)(x)=\epsilon_{B}\left(\delta_{B}(f)\right)(x)
\end{aligned}
$$

When a series of openings with SE of increasing size $(\lambda)$ are sequentially computed on the image, a morphological opening pyramid is obtained and it can be defined as:

$$
\Pi_{\gamma}(f)=\left\{\Pi_{\gamma \lambda}: \Pi_{\gamma \lambda}=\gamma_{\lambda}(f), \forall \lambda \in\left[0, \ldots, n_{\max }\right]\right\}
$$

where $n_{\max }$ represents the maximum size of the structuring element. By duality, the morphological closing pyramid $\Pi_{\varphi}$ is defined in the same way.

A shape descriptor can be defined using the morphological pyramids above described. Let $m(f)$ be the Lebesge measure of a discrete image $f$. The granulometry curve, or pattern spectrum of $f$ with respect to $\Gamma$ is defined as:

$$
P S_{\Gamma}(f, n)=P S(f, n)=\frac{m\left(\Pi_{\gamma n}(f)\right)-m\left(\Pi_{\gamma n+1}(f)\right)}{m(f)}, n \geq 0
$$

By duality, this concept extends to the anti-granulometry curve $P S_{\Phi}(f)$.

Granulometry is used in this work by applying a series of morphological opening (closing) operations with increasing-size structuring elements (SE) defined by a specific step $(s=2)$ and a maximum value $\left(n_{\max }=22\right)$, to obtain a local description of the shape and size of the retinal exudates. SE is a disk or a line giving place to an isotropic or angular granulometry, respectively. In the end, four feature vectors are obtained: two regarding the isotropic granulometry (opening and closing operations) and two regarding the angular granulometry. Thus, these pattern spectrums are combined giving place to the morphological feature vector composed by 44 elements. Note that angular granulometry is computed in the directions $0^{\circ}, 45^{\circ}, 90^{\circ}$ and $135^{\circ}$. The regions containing optic disk pixels are not considered during the feature extraction stage. To detect the optic disk, the method proposed in [22] is used. Figure 4 illustrates the results obtained by applying this method.

\subsection{Retinal Tissue Classification}

After the feature extraction step, the Support Vector Machines (SVM) classifier [23] is used to classify each region in healthy or pathological. This classifier generates a classification model, based on the information of features and labels 


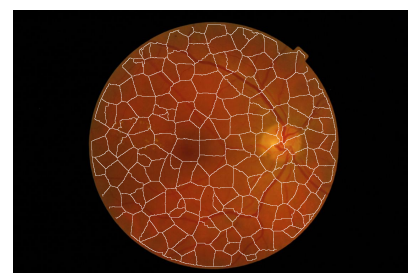

(a)

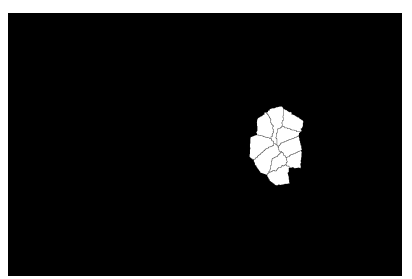

(b)

Fig. 4. Optic disk detection results. (a) Waterpixels, (b) Optic disk regions.

of the training set, able to predict the correct class of new samples belonging to the test set. With this purpose, SVM maximises the distance between the hyperplanes defined by the support vectors with the aim of finding the optimal separation between classes. The Radial Basis Function (RBF) kernel is employed when the SVM algorithm is applied. The library for support vector machines (libSVM) used was introduced by C. Chang in [23].

An external $K$-fold cross validation is performed to provide robustness to the decision system and to guarantee that all the instances are used in the creation of the model and in the prediction stage. A different partition is selected as test subset while the rest of the partitions are used to train the model.

As it was previously discussed, exudates represent only a small percentage of the total number of pixels that compose the retinal image, which results in a very unbalanced dataset when the feature extraction is performed. Training the classifier with this dataset can result in an overfitting to the class "healthy".

To avoid this problem, the set of all healthy samples is randomly permuted and partitioned into $T=\operatorname{round}(M / N)$ subsets, being $M$ and $N$ the number of healthy and pathological samples, respectively. After that, a set of $T$ classifiers is trained using all pathological training samples and each partition of healthy training samples. Finally, during the test stage, testing samples are evaluated for each of the $T$ models and soft majority voting is applied to the output probabilities as the final criterion. If the obtained probability is higher than a given threshold $(\delta)$, the region is assigned to the class "pathological".

\section{$3 \quad$ Results}

The database chosen to validate the method proposed in this project was the E-OPHTHA public database [24]. This database is divided in two subsets and the one used in this paper is composed by 47 images containing exudates. These lesions are manually annotated by experts. It was necessary to perform a spatial normalization of the fundus images since they presented different resolutions.

Two different strategies to obtain waterpixels were presented in this paper. These regions were used to perform a local feature extraction in fundus images for detecting pathological areas. To evaluate these strategies, the procedure explained in section 2.3. was used to train different classification models. 
In order to compare the strategies proposed in this paper with another stateof-the-art method, the same procedure was applied using the SLIC algorithm to generate superpixels. This algorithm adapts a $k$-means clustering approach to efficiently generate superpixels. By default, the only parameter of the algorithm is $k$, which is the desired number of approximately equally sized superpixels. Several tests using the original images (OI) and the images without the blood vessels $(\mathrm{W} / \mathrm{V})$ were performed for each of the strategies mentioned. The images without blood vessels were obtained through the inpainting technique [25].

Table 1 contains the Area Under ROC Curve (AUC), accuracy, sensitivity and specificity (as well as the standard deviation associated with each of these metrics) of the resulting classification processes, taking into account the groundtruth provided by the ophthalmologists. The results of accuracy, sensitivity and specificity were obtained using a decision threshold of $\delta=0.5$.

As it can be observed through the analysis of Table 1, the values of accuracy improve, for each of regions of interested developed, when the inpainting technique is applied in order to remove the blood vessels from the original images. During the evaluation of the different techniques it is important to remember the importance of obtaining a good trade-off between the sensitivity and specificity. Additionally, in the context of this paper, sensitivity plays a major role since this measure represents the ability of our system to detect exudates. Having that in mind, and through the analysis of Table 1, it is possible to conclude that the waterpixels approaches outperform the SLIC [18] method when it comes to generate the most appropiate regions to detect exudates.

The ROC curves for each of the tests performed with the images without vessels are represented in Fig. 5. As it can be observe, the c-Waterpixels outperform the other methods for lower false positive rates while the $\mathrm{m}$-waterpixels exceed the c-Waterpixels and SLIC superpixels for highest false positives rates.

Table 1. AUC, accuracy, sensitivity and specificity related to the exudate detection on the original images (OI) and images without vessels $(\mathrm{W} / \mathrm{V})$ for each of the region of interest developed: m-Waterpixels, c-Waterpixels and SLIC superpixels.

\begin{tabular}{ccc|cc|cc} 
& \multicolumn{2}{c}{ m-Waterpixels } & \multicolumn{2}{c}{ c-Waterpixels } & \multicolumn{2}{c}{ SLIC Superpixels } \\
\cline { 2 - 7 } & OI & W/V & OI & W/V & OI & W/V \\
\cline { 2 - 7 } AUC & $0.7998 \pm 0.0317$ & $\mathbf{0 . 8 2 8 7} \pm \mathbf{0 . 0 2 4 6}$ & $0.7937 \pm 0.0325$ & $0.8277 \pm 0.0199$ & $0.7987 \pm 0.0311$ & $0.8169 \pm 0.0299$ \\
Accuracy & $0.6514 \pm 0.1093$ & $0.7585 \pm 0.0676$ & $0.6650 \pm 0.0870$ & $0.7545 \pm 0.0679$ & $0.7635 \pm 0.0238$ & $\mathbf{0 . 8 0 3 7} \pm \mathbf{0 . 0 2 2 2}$ \\
Sensitivity & $\mathbf{0 . 7 7 7 9} \pm \mathbf{0 . 0 9 8 7}$ & $0.7216 \pm 0.0834$ & $0.7651 \pm 0.0477$ & $0.7496 \pm 0.0295$ & $0.6567 \pm 0.0824$ & $0.6656 \pm 0.0634$ \\
Specificity & $0.6363 \pm 0.1295$ & $0.7595 \pm 0.0789$ & $0.6546 \pm 0.0990$ & $0.7539 \pm 0.0757$ & $0.7736 \pm 0.0305$ & $\mathbf{0 . 8 1 7 5} \pm \mathbf{0 . 0 2 7 3}$
\end{tabular}

\section{Conclusions}

In this paper, a system based on local feature extraction and SVM classification is used to develop and compare different strategies of exudates automated detection. These strategies involve performing the local feature extraction using three different methods to generate non-uniform regions: m-Waterpixels, c-Waterpixels and SLIC superpixels. Analysing the results obtained for each of the proposed methods, it is possible to conclude that the watershed-based approaches lead to 


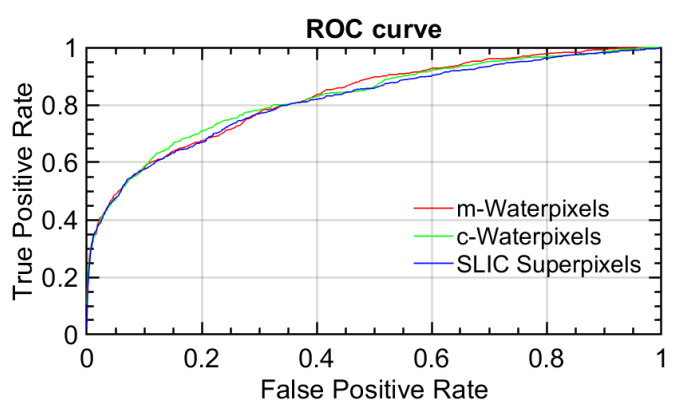

Fig. 5. ROC curves for the different superpixels methods.

a better detection of pathological areas. The results also demonstrate that applying the inpainting technique to remove the blood vessels is essential to obtain a more accurate detection of exudates. Future work will allow the detection of other lesions related to DR, such as microaneurisms and hemorrhages.

Acknowledgements. This paper was supported by the the European Union's Horizon 2020 research and innovation programme under the Project GALAHAD [H2020-ICT-2016-2017, 732613]. The work of Adrián Colomer has been supported by the Spanish Government under a FPI Grant [BES-2014-067889]. We gratefully acknowledge the support of NVIDIA Corporation with the donation of the Titan Xp GPU used for this research.

\section{References}

1. Sidibé, D., Sadek, I., Mériaudeau, F.: Discrimination of retinal images containing bright lesions using sparse coded features and svm. Computers in biology and medicine 62 (2015) 175-184

2. Zhou, W., Wu, C., Yi, Y., Du, W.: Automatic detection of exudates in digital color fundus images using superpixel multi-feature classification. IEEE Access $\mathbf{5}$ (2017) 17077-17088

3. Sinthanayothin, C., Boyce, J.F., Williamson, T.H., Cook, H.L., Mensah, E., Lal, S., Usher, D.: Automated detection of diabetic retinopathy on digital fundus images. Diabetic medicine 19(2) (2002) 105-112

4. Walter, T., Klein, J.C., et al.: A contribution of image processing to the diagnosis of diabetic retinopathy-detection of exudates in color fundus images of the human retina. IEEE transactions on medical imaging 21(10) (2002) 1236-1243

5. Ali, S., Sidibé, D., Adal, K.M., Giancardo, L., Chaum, E., Karnowski, T.P., Mériaudeau, F.: Statistical atlas based exudate segmentation. Computerized Medical Imaging and Graphics 37(5-6) (2013) 358-368

6. Zhang, X., Thibault, G., Decencière, E., Marcotegui, B., et al.: Exudate detection in color retinal images for mass screening of diabetic retinopathy. Medical image analysis 18(7) (2014) 1026-1043

7. Li, H., Chutatape, O.: Automated feature extraction in color retinal images by a model based approach. IEEE Transactions on biomedical engineering 51(2) (2004) $246-254$ 
8. Welfer, D., Scharcanski, J., Marinho, D.R.: A coarse-to-fine strategy for automatically detecting exudates in color eye fundus images. computerized medical imaging and graphics 34(3) (2010) 228-235

9. Giancardo, L., Meriaudeau, F., Karnowski, T.P., Li, Y., Garg, S., Tobin, K.W., Chaum, E.: Exudate-based diabetic macular edema detection in fundus images using publicly available datasets. Medical image analysis 16(1) (2012) 216-226

10. Amel, F., Mohammed, M., Abdelhafid, B.: Improvement of the hard exudates detection method used for computer-aided diagnosis of diabetic retinopathy. International Journal of Image, Graphics and Signal Processing 4(4) (2012) 19

11. Akram, M.U., Khalid, S., Tariq, A., Khan, S.A., Azam, F.: Detection and classification of retinal lesions for grading of diabetic retinopathy. Computers in biology and medicine 45 (2014) 161-171

12. Akram, M.U., Tariq, A., Khan, S.A., Javed, M.Y.: Automated detection of exudates and macula for grading of diabetic macular edema. Computer methods and programs in biomedicine 114(2) (2014) 141-152

13. Machairas, V.: Waterpixels and their application to image segmentation learning. $\mathrm{PhD}$ thesis, Université de recherche Paris Sciences et Lettres (2016)

14. Shi, J., Malik, J.: Normalized cuts and image segmentation. IEEE Transactions on pattern analysis and machine intelligence 22(8) (2000) 888-905

15. Veksler, O., Boykov, Y., Mehrani, P.: Superpixels and supervoxels in an energy optimization framework. Computer Vision-ECCV 2010 (2010) 211-224

16. Comaniciu, D., Meer, P.: Mean shift: A robust approach toward feature space analysis. IEEE Transactions on pattern analysis and machine intelligence 24(5) (2002) 603-619

17. Levinshtein, A., Stere, A., Kutulakos, K.N., Fleet, D.J., Dickinson, S.J., Siddiqi, K.: Turbopixels: Fast superpixels using geometric flows. IEEE transactions on pattern analysis and machine intelligence 31(12) (2009) 2290-2297

18. Achanta, R., Shaji, A., Smith, K., Lucchi, A., Fua, P., Süsstrunk, S.: Slic superpixels compared to state-of-the-art superpixel methods. IEEE transactions on pattern analysis and machine intelligence 34(11) (2012) 2274-2282

19. Machairas, V., Faessel, M., Cárdenas-Peña, D., Chabardes, T., Walter, T., Decencière, E.: Waterpixels. IEEE Transactions on Image Processing 24(11) (2015) 3707-3716

20. Ojala, T., Pietikainen, M., Maenpaa, T.: Multiresolution gray-scale and rotation invariant texture classification with local binary patterns. IEEE Transactions on pattern analysis and machine intelligence 24(7) (2002) 971-987

21. Guo, Z., Zhang, L., Zhang, D.: Rotation invariant texture classification using lbp variance (lbpv) with global matching. Pattern recognition 43(3) (2010) 706-719

22. Morales, S., Naranjo, V., Angulo, J., Alcañiz, M.: Automatic detection of optic disc based on pca and mathematical morphology. IEEE transactions on medical imaging 32(4) (2013) 786-796

23. Chang, C.C., Lin, C.J.: Libsvm: a library for support vector machines. ACM transactions on intelligent systems and technology (TIST) 2(3) (2011) 27

24. Decencière, E., Cazuguel, G., Zhang, X., Thibault, G., Klein, J.C., Meyer, F., et al.: Teleophta: Machine learning and image processing methods for teleophthalmology. Irbm 34(2) (2013) 196-203

25. DErrico, J.: inpaint_nans (http://kr. mathworks. com/matlabcentral/fileexchange/4551-inpaint-nans), matlab central file exchange. Retrieved Aug 13 (2004) 2012 